\title{
A NOTE ON THE PLANE VERTICAL WAVEMAKER IN THE PRESENCE OF SURFACE TENSION
}

\author{
BY \\ B. N. MANDAL AND S. BANDYOPADHYAY \\ Indian Statistical Institute, Calcutta, India
}

\begin{abstract}
This paper presents another method for finding the known wave motion due to a vertical wavemaker in the presence of surface tension, either for finite constant or infinite depth, by using the Fourier integral transform technique and suitably exploiting the regularity condition of the transform.
\end{abstract}

1. Introduction. The problem of forced surface waves produced by a timeharmonic plane vertical wavemaker was treated in 1929 by Havelock [1] within the framework of linearized theory of water waves assuming the irrotational motion of the liquid. Later Rhodes-Robinson [2] extended this classical wavemaker problem to include the effect of surface tension at the free surface and used a method based on the application of Green's integral theorem in the fluid domain to the potential function and a suitably chosen Green's function to obtain the solution of the resulting mixed boundary value problem. Recently Mandal and Chakrabarti [3] solved the two-dimensional plane vertical wavemaker problem in deep water (when the effect of surface tension at the free surface is neglected) using the Fourier integral transform technique and suitably exploiting a regularity condition of the transform. However, the extension to include the effect of surface tension requires considerably more detailed analysis.

In the present paper the plane vertical wavemaker problem in the presence of surface tension is reinvestigated for the two cases of infinite and finite constant depth of fluid by using the Fourier transform method in the horizontal direction. This reduces the problem to an ordinary differential equation on an interval over the depth, for which the solution is known. The outgoing waves are obtained separately using a regularity condition. The solution depends on a parameter $\lambda$ in the edge condition that is regarded as known. However, it is not true that $\lambda$ is known; as was supposed earlier. Recent studies by Hocking [4] show that a dynamical edge condition must be satisfied, and from this $\lambda$ can be determined using this solution.

2. Formulation of the problem. We consider the irrotational motion of an incompressible inviscid fluid due to a harmonically oscillating vertical plane wavemaker under the action of gravity and surface tension. We use a rectangular cartesian

Received February 27, 1989.

(C)1991 Brown University 
coordinate system in which the $y$-axis is taken vertically downwards so that $y=0$, $x>0$ is the undisturbed free surface and $x=0$ is the wavemaker, and in the undisturbed state the fluid occupies the region $x>0$ and $y \geq 0$ or $0 \leq y \leq h$ according as it is of infinite depth or uniform finite depth $h$. The motion is two-dimensional and time-harmonic and is described by a velocity potential $\varphi(x, y, t)$ which is the real part of $\Psi(x, y, t) e^{-i \sigma t}, \sigma$ being the circular frequency. Then $\Psi(x, y)$ satisfies

$$
\nabla^{2} \Psi=0 \text { in the fluid region; }
$$

the linearized free surface condition

$$
K \Psi+\Psi_{y}+M \psi_{y y y}=0 \quad \text { on } y=0, x>0,
$$

where $K=\sigma^{2} / g$ and $M=T / \rho g, T$ being the surface tension, $\rho$ the density, and $g$ the gravity; the wavemaker condition

$$
\Psi_{x}=u(y) \quad \text { on } x=0
$$

the bottom condition

$$
\nabla \Psi \rightarrow 0 \quad \text { as } y \rightarrow \infty \text { for fluid of infinite depth },
$$

or

$$
\Psi_{y}=0 \quad \text { on } y=h \text { for fluid of uniform finite depth } h \text {; }
$$

and the condition that it represents outgoing waves at large distances from the wavemaker, which can be mathematically expressed as

$$
\Psi \rightarrow c e^{-k_{0} y+i k_{0} x} \quad \text { as } x \rightarrow \infty,
$$

where, in the case of fluid of infinite depth, $k=k_{0}$ is the unique positive real root of

$$
K-\left(1+M k^{2}\right) k=0,
$$

or as

$$
\Psi \rightarrow c_{0} \cosh \alpha_{0}(h-y) e^{i \alpha_{0} x} \quad \text { as } x \rightarrow \infty,
$$

where, in the case of fluid of uniform finite depth $h, k=\alpha_{0}$ is the unique positive real root of the equation

$$
k\left(1+M k^{2}\right) \sinh k h-K \cosh k h=0 .
$$

It may be noted that the constants $c$ and $c_{0}$ in (2.6) and (2.8) are unknown and are to be determined. These will be determined by using a regularity condition of the Fourier transform in the following sections.

Finally the edge condition prescribing the free surface slope at the wavemaker as derived by Evans [5] in connection with the effect of surface tension on the waves produced by a circular cylinder and used later by Rhodes-Robinson [2] is given by

$$
\Psi_{x y}(o+, o)=\lambda
$$

where $\lambda$ may be regarded as known. However, see the Introduction for a brief discussion on $\lambda$. 
3. Solution for fluid of infinite depth. Let us write

$$
\Psi=c e^{-k_{0} y+i k_{0} x}+\Phi(x, y) ;
$$

then $\Phi(x, y)$ satisfies

$$
\begin{aligned}
& \nabla^{2} \Phi=0, \quad y>0, x>0, \\
& K \Phi+\Phi_{y}+M \Phi_{y y y}=0 \quad \text { on } y=0, x>0 \\
& \Phi_{x}=v(y) \quad \text { on } x=0, y>0 \\
& \nabla \Phi \rightarrow 0 \text { as } y \rightarrow \infty \\
& \Phi \rightarrow 0 \text { as } x \rightarrow \infty \\
& \Phi_{x y}(o+, o)=\lambda+i k_{0}^{2} c
\end{aligned}
$$

where

$$
v(y)=u(y)-i c k_{0} e^{-k_{0} y} .
$$

Let

$$
\chi(y ; \xi)=\int_{0}^{\infty} \Phi(x, y) \cos \xi x d x
$$

then $x$ satisfies

$$
\begin{aligned}
& \chi_{y y}-\xi^{2} \chi=v(y), \quad y>0, \\
& K \chi+\chi_{y}\left(1+M \xi^{2}\right)+M\left(\lambda+i k_{0}^{2} c\right)=0 \quad \text { on } y=0, \\
& \chi_{y} \rightarrow 0 \quad \text { as } y \rightarrow \infty .
\end{aligned}
$$

The solution of (3.4) is given by (cf. Mikhlin [6])

$$
\begin{aligned}
\chi(y ; \xi)= & -\int_{0}^{\infty} G(y, s)\left\{v(s)-\frac{M}{K}\left(\lambda+i k_{0}^{2} c\right) \xi^{2}\right\} d s \\
& -\frac{M}{k}\left(\lambda+i k_{0}^{2} c\right),
\end{aligned}
$$

where $G(y, s)$ is the associated Green's function defined by

$$
G(y, s)=\frac{e^{-\xi s}\left\{\left(1+M \xi^{2}\right) \xi \cosh \xi y-K \sinh \xi y\right\}}{\xi\left\{\left(1+M \xi^{2}\right) \xi-K\right\}}
$$

for $0 \leq y \leq s$. (For $0 \leq s \leq y, y$ and $s$ are to be interchanged in (3.6).) Using (3.6) and (3.2) in (3.5) we obtain

$$
\begin{aligned}
\chi(y ; \xi)= & \frac{\left(1+M \xi^{2}\right) \xi \cosh \xi y-K \sinh \xi y}{\left\{\left(1+M \xi^{2}\right) \xi-K\right\} \xi} \\
& \times\left[\frac{i c k_{0}}{k_{0}+\xi} \frac{M}{K}\left(\lambda+i k_{0}^{2} c\right)-a(\xi)\right]-\frac{M}{K}\left(\lambda+i k_{0}^{2} c\right),
\end{aligned}
$$

where

$$
a(\xi)=\int_{0}^{\infty} u(s) e^{-\xi s} d s .
$$

Now $\chi(y ; \xi)$ is the Fourier cosine transform of a certain function, and treated as a function of the complex variable $\xi$, it cannot have a singularity on the real axis. 
This immediately suggests that the unknown constant $c$ appearing in (3.7) must be such that $\chi(y ; \xi)$ is regular at the point $\xi=k_{0}$. This gives

$$
c=-2 i \frac{K a\left(k_{0}\right)-k_{0} M \lambda}{k_{0}\left(1+3 M k_{0}^{2}\right)} \text {. }
$$

The Fourier cosine inversion formula gives $\Phi(x, y)$ as

$$
\Phi(x, y)=\frac{2}{\pi} \int_{0}^{\infty} \chi(\xi, y) \cos \xi x d \xi,
$$

where $\chi(\xi, y)$ is given in (3.7).

Writing $2 \cos \xi x$ as $e^{i \xi x}+e^{-i \xi x}$ in (3.10) and rotating the contour along the positive imaginary axis for the integral involving $e^{i \xi x}$ and along the negative imaginary axis for the integral involving $e^{-i \xi x}$ we obtain

$$
\begin{aligned}
\Phi(x, y)=-\frac{2}{\pi} \int_{0}^{\infty} & \frac{\left(1-M k^{2}\right) k \cos k y-K \sin k y}{\left(1-M k^{2}\right)^{2} k^{2}+K^{2}} \\
& \times\left[-M \lambda+\int_{0}^{\infty} \frac{\left(1-M k^{2}\right) k \cos k s-K \sin k s}{k} u(s) d s\right] e^{-k x} d k,
\end{aligned}
$$

where we have employed (3.8). Hence $\Psi(x, y)$ is found and we finally obtain

$$
\begin{aligned}
\varphi(x, y)= & \operatorname{Re}\left\{\Psi(x, y) e^{-i \sigma t}\right\} \\
= & \frac{2}{\pi} \cos \sigma t \int_{0}^{\infty} \frac{b(k) e^{-k x}\left\{\left(1-M k^{2}\right) k \cos k y-K \sin k y\right\}}{k^{2}\left(1-M k^{2}\right)^{2}+K^{2}} d k \\
& -\frac{2 B}{1+3 M k_{0}^{2}} e^{-k_{0} y} \sin \left(k_{0} x-\sigma t\right),
\end{aligned}
$$

where

$$
\begin{aligned}
& b(k)=-\frac{1}{k} \int_{0}^{\infty}\left\{k\left(1-M k^{2}\right) \cos k s-K \sin k s\right\} u(s) d s+M \lambda, \\
& B=-\left(1+M k_{0}^{2}\right) \int_{0}^{\infty} u(s) e^{-k_{0} s} d s+M \lambda .
\end{aligned}
$$

(3.12) coincides with the real part of the expression given in [2] obtained by a different technique.

4. Solution for fluid of finite depth. In this case we write

$$
\Psi(x, y)=c_{0} \frac{\cosh \alpha_{0}(h-y)}{\cosh \alpha_{0} h} e^{i \alpha_{0} x}+\Phi(x, y) ;
$$


then $\Phi(x, y)$ satisfies

$$
\begin{aligned}
& \nabla^{2} \Phi=0, \quad 0<y<h, \quad x>0, \\
& K \Phi+\Phi_{y}+M \Phi_{y y y}=0 \quad \text { on } y=0, x>0 \\
& \Phi_{x}=v(y) \quad \text { on } x=0,0<y<h, \\
& \Phi_{y}=0 \quad \text { on } y=h, \quad x>0, \\
& \Phi \rightarrow 0 \quad \text { as } x \rightarrow \infty, \\
& \Phi_{x y}(o+, o)=\lambda+i \alpha_{0}^{2} c_{0} \tanh \alpha_{0} h
\end{aligned}
$$

where

$$
v(y)=u(y)-i \alpha_{0} c_{0} \frac{\cosh \alpha_{0}(h-y)}{\cosh \alpha_{0} h} .
$$

Defining as before

$$
\chi(y ; \xi)=\int_{0}^{\infty} \Phi(x, y) \cos \xi x d x, \quad 0<y<h
$$

we see that $\chi$ satisfies

$$
\begin{aligned}
& \chi_{y y}-\xi^{2} \chi=v(y), \quad 0<y<h, \\
& K \chi+\chi_{y}\left(1+M \xi^{2}\right)+M\left(\lambda+i \alpha_{0}^{2} c_{0} \tanh \alpha_{0} h\right)=0 \quad \text { on } y=0 \\
& \chi_{y}=0 \quad \text { on } y=h .
\end{aligned}
$$

Its solution is

$$
\begin{aligned}
\chi(\xi ; y)= & -\int_{0}^{h} G(y, s)\left[v(s)-\frac{M}{K}\left(\lambda+i \alpha_{0}^{2} c_{0} \tanh \alpha_{0} h\right) \xi^{2}\right] d s \\
& -\frac{M}{K}\left(\lambda+i \alpha_{0}^{2} c_{0} \tanh \alpha_{0} h\right)
\end{aligned}
$$

where $G(y, s)$ is now given by

$$
G(y, s)=\frac{\cosh \xi(h-s)}{\xi \Delta(\xi)}\left[\xi\left(1-M \xi^{2}\right) \cosh \xi y-K \sinh \xi y\right]
$$

for $0 \leq y \leq s \leq h$, where

$$
\Delta(\xi)=\xi\left(1+M \xi^{2}\right) \sinh \xi h-K \cosh \xi h .
$$

(For $0 \leq s \leq y \leq h, y$ and $s$ in (4.5) are to be interchanged.) We note that $\Delta(\xi)$ has two real zeros at $\xi= \pm \alpha_{0}$ and has an infinite number of imaginary zeros at $\xi= \pm i k_{n}, n=1,2, \ldots$, where the $k_{n}$ 's satisfy (cf. Rhodes-Robinson [2])

$$
k_{n}\left(1-M k_{n}^{2}\right) \sin k_{n} h+K \cos k_{n} h=0
$$


Now (4.4) can be rewritten as

$$
\begin{aligned}
\chi(y ; \xi)= & \frac{K \sinh \xi y-\xi\left(1+M \xi^{2}\right) \cosh \xi y}{\xi \Delta(\xi)} \\
& \times\left[a(\xi)-\frac{i c \alpha_{0}}{\xi^{2}-\alpha_{0}^{2}}\left(\xi \sinh \xi h-\frac{K}{1+M \alpha_{0}^{2}} \cosh \xi h\right)\right. \\
& \left.-\frac{M}{K}\left(\lambda+i \alpha_{0}^{2} c_{0} \tanh \alpha_{0} h\right) \xi \sinh \xi h\right] \\
& -\frac{M}{K}\left(\lambda+i \alpha_{0}^{2} c_{0} \tanh \alpha_{0} h\right),
\end{aligned}
$$

where

$$
a(\xi)=\int_{0}^{\infty} u(s) \cosh \xi(h-s) d s
$$

As before, using the argument that $\chi(y ; \xi)$, regarded as a function of $\xi$, cannot have a singularity on the real axis, we determine the unknown constant $c_{0}$ as

$$
c_{0}=-\frac{4 i \cosh \alpha_{0} h\left\{a\left(\alpha_{0}\right)\left(1+M \alpha_{0}^{2}\right)-M \lambda \cosh \alpha_{0} h\right\}}{2 \alpha_{0} h\left(1+M \alpha_{0}^{2}\right)+\left(1+3 M \alpha_{0}^{2}\right) \sinh 2 \alpha_{0} h} .
$$

Employing the inversion formula for the cosine transform we obtain $\Phi(x, y)$ as

$$
\Phi(x, y)=\frac{2}{\pi} \int_{0}^{\infty} \chi(y ; \xi) \cos \xi x d \xi,
$$

where $\chi(y ; \xi)$ is now given by (4.6).

The contour in (4.9) can be extended to the whole real axis with $2 \cos \xi x$ replaced by $e^{i \xi x}$. This may then be evaluated by the method of residues at the poles $k=i k_{n}$, $n=1,2, \ldots$. Note that there are no singularities at $\xi= \pm \alpha_{0}$. Thus,

$$
\begin{aligned}
\Phi(x, y)=\sum_{n} & \frac{4 \pi \cos k_{n}(h-y) \cos k_{n} h}{2 k_{n} h\left(1-M k_{n}^{2}\right)+\left(1-3 M k_{n}^{2}\right) \sin 2 k_{n} h} \\
& \times\left[-\frac{1-M k_{n}^{2}}{\cos k_{n} h} \int_{0}^{h} u(s) \cos k_{n}(h-s) d s+M \lambda\right] e^{-k_{n} x},
\end{aligned}
$$

so that we finally obtain

$$
\begin{aligned}
\varphi(x, y)= & \frac{-4 \sin \left(\alpha_{0} x-\sigma t\right) \cosh \alpha_{0} h \cosh \alpha_{0}(h-y) A_{0}}{2 \alpha_{0} h\left(1+M \alpha_{0}^{2}\right)+\left(1+3 M \alpha_{0}^{2}\right) \sinh 2 \alpha_{0} h} \\
& +4 \pi \cos \sigma t \sum_{n} \frac{A_{n} \cos k_{n} h \cos k_{n}(h-y) e^{-k_{n} x}}{2 k_{n} h\left(1-M k_{n}^{2}\right)+\left(1-3 M k_{n}^{2}\right) \sin 2 k_{n} h},
\end{aligned}
$$

where

$$
\begin{aligned}
& A_{0}=-\frac{1+M \alpha_{0}^{2}}{\cosh \alpha_{0} h} \int_{0}^{h} u(s) \cosh \alpha_{0}(h-s) d s+M \lambda, \\
& A_{n}=-\frac{1-M k_{n}^{2}}{\cos k_{n} h} \int_{0}^{h} u(s) \cos k_{n}(h-s) d s+M \lambda .
\end{aligned}
$$

(4.11) coincides with the real part of the expression given in [2] obtained by a different technique. 
5. Conclusion. The Fourier transform technique is used to solve the classical wavemaker problem in the presence of surface tension at the free surface for the two cases of infinite and finite constant depth of fluid. By exploiting a regularity condition of the transform the outgoing waves are obtained separately. The method seems to be quite simple in comparison with the somewhat complicated method based on Green's integral theorem used by Rhodes-Robinson [2].

Acknowledgment. The authors take this opportunity to thank the referee for his comments and suggestions to improve the paper and for drawing their attention to the reference [4] in connection with the edge condition. This work was supported by the Third World Academy of Sciences, ICTP, Trieste, through research grant TWASRG NO. 274.

\section{REFERENCES}

[1] T. H. Havelock, Forced surface waves on water, Philos. Mag. 8, 569-576 (1929)

[2] P. F. Rhodes-Robinson, On the forced surface waves due to a vertical wave maker in the presence of a surface tension, Proc. Cambridge Philos. Soc. 70, 323-337 (1971)

[3] B. N. Mandal and A. Chakrabarti, The plane vertical wavemaker problem-revisited, Appl. Math. Lett. 1, 255-258 (1988)

[4] L. M. Hocking, Capillary-gravity waves produced by a heaving body, J. Fluid Mech. 186, 337-349 (1988)

[5] D. V. Evans, The effect of surface tension on the waves produced by a heaving circular cylinder, Proc. Cambridge Philos. Soc. 64, 833-847 (1968)

[6] S. G. Mikhlin, Integral Equations, Pergamon Press, 1964, p. 280 